\title{
MARIE-ÈVE THÉRENTY, Femmes de presse, femmes de lettre. De Delphine de Girardin à Florence Aubenas
}

\section{Gabriella Bosco}

\section{(2) OpenEdition}

\section{Journals}

\section{Edizione digitale}

URL: https://journals.openedition.org/studifrancesi/32456

DOI: 10.4000/studifrancesi.32456

ISSN: 2427-5856

\section{Editore}

Rosenberg \& Sellier

\section{Edizione cartacea}

Data di pubblicazione: 1 août 2020

Paginazione: 458-459

ISSN: 0039-2944

\section{Notizia bibliografica digitale}

Gabriella Bosco, «MARIE-ĖVe thérenty, Femmes de presse, femmes de lettre. De Delphine de Girardin à Florence Aubenas», Studi Francesi [Online], 191 (LXIV | II) | 2020, online dal 01 septembre 2020, consultato il 18 septembre 2021. URL: http://journals.openedition.org/studifrancesi/32456 ; DOI: https://doi.org/10.4000/studifrancesi.32456

Questo documento è stato generato automaticamente il 18 septembre 2021.

\section{(c) $($ ) $(9)$}

Studi Francesi è distribuita con Licenza Creative Commons Attribuzione - Non commerciale - Non opere derivate 4.0 Internazionale. 


\title{
MARIE-ÈVE THÉRENTY, Femmes de presse, femmes de lettre. De Delphine de Girardin à Florence Aubenas
}

\author{
Gabriella Bosco
}

\section{NOTIZIA}

MARIE-ÈVE THÉRENTY, Femmes de presse, femmes de lettre. De Delphine de Girardin à Florence Aubenas, CNRS Editions, Paris, 2019, 396 pp.

1 Dedicato alle donne che hanno fatto del giornalismo il loro mestiere, il volume di Marie-Ève THÉRENTY colma finalmente una mancanza ingiustificata, nell'ambito della storia di quel genere di scrittura che solo per convenzione è stato a lungo considerato appannaggio degli uomini, mentre fin dalle origini non poche e di valore sono le donne che l'hanno praticato.

Dall'inizio del xIX secolo fino ad alcuni grandi firme novecentesche, l'A. offre un panorama del giornalismo "altro" che le donne hanno saputo imporre, senza timore di invadere campi come la politica, la cronaca giudiziaria, quella sportiva o il grande reportage, ovvero forme per pregiudizio di antica data riservate ai colleghi maschi. Proprio allo scopo di attribuire a ognuna delle giornaliste studiate il giusto spazio accanto a coloro che tutti i manuali del giornalismo riportano, Marie-Ève Thérenty ha voluto proporre una storia alternativa, programmaticamente complementare rispetto a quella tradizionale.

3 L'impostazione data dall'A., che è docente di Letteratura francese all'Université PaulValéry Montpellier 3 specializzata in giornalismo, pone come premessa la specificità del tipo di scrittura studiata, che è attività sociale a molteplici livelli, qualificandosi innanzitutto come testimonianza, restituzione di un determinato rapporto con il reale; ed è inoltre occupazione economica, retribuita, che viene esercitata nel quadro di un'impresa. In quanto tale, a maggior ragione nei secoli passati, la donna che vi si è 
dedicata si è trovata in condizioni notevolmente differenziate rispetto all'uomo. A partire dall'educazione ricevuta che a lungo non ha consentito la stessa copertura disciplinare. Marie- Ève Thérenty ricorda che ancora nel 1880 la legge Camille Sée, regolando i contenuti dell'insegnamento secondario femminile, non proponeva corsi di filosofia, suggeriva un'iniziazione basica nelle materie scientifiche mentre disponeva insegnamenti di ordine comportamentale, di economia domestica e cucito. E non era naturalmente previsto l'accesso all'università. Solo nel 1924 la legge Bérard giunse alla parziale assimilazione dell'insegnamento femminile a quello maschile con programmi comuni in francese, lingue straniere, scienze, storia, geografia e disegno. Ma anche allora, la legge continuava a non garantire alle donne diritti rapportabili a quelli degli uomini, in fatto di scelta del mestiere o indipendenza delle risorse. In particolare l'attività giornalistica era inibita proprio da questo punto di vista, richiedendo libertà d'azione, di tempo e di rapporto con il mondo. A lungo, la presenza della donna in certi ambienti e luoghi pubblici come le università, i luoghi politici o le accademie è stata di natura subalterna. Condizione strettamente legata, prima del 1944, alla mancanza del diritto di voto, che comportava de facto l'esclusione della donna dalla possibilità di farsi eleggere in ruoli importanti nella sfera pubblica.

4 Tale premessa metodologica ha comportato la scelta di occuparsi dei grandi giornali, dei settimanali d'informazione e delle grandi riviste, e non di stampa femminile o femminista, campi in cui gli studi non sono peraltro mancati. L'A. ha ritenuto più interessante seguire il percorso delle donne all'interno della stampa generalista.

Data d'avvio dell'analisi è il 1836, anno di fondazione da parte di Émile de Girardin del giornale La Presse e da parte di sua moglie Delphine de Girardin della creazione di uno dei generi giornalistici più fecondi e rappresentativi del XIX secolo, la cronaca parigina. L'inchiesta giunge fino al 1944, ma include alcuni grandi nomi successivi - quelli di Françoise Giroud, Marguerite Duras e Florance Aubenas - per illustrare i cambiamenti determinati nel campo del mestiere giornalistico dall'acquisizione del diritto al voto, che di fatto attenua, pur senza farle scomparire del tutto, le differenze di genere.

La scommessa all'origine del volume è stata inoltre quella di dimostrare che le donne hanno accentuato la tendenza della stampa del xIX secolo a farsi letteraria. Per far entrare la loro prosa nei giornali, le donne hanno in genere puntato sulla finzionalizzazione, o sulla maschera fornita dal lirismo. Ma altresì è stata determinante la volontà di far luce sulla grande quantità di pratiche giornalistiche messe in atto dalle donne, a esclusione - precisa l'A. nelle battute conclusive del suo Avant-propos (pp. 9-20) - del genere della critica, sia letteraria che teatrale o musicale, che avrebbe richiesto un altro tipo di problematizzazione rispetto all'attualità politica, sociale o culturale.

7 La suddivisione in capitoli, cronologici, vede avvicendarsi modelli giornalistici diversi per i quali, di volta in volta, l'A. ha utilizzato il ricorso a una figura mitologica o letteraria. Così, il primo è dedicato a Les chroniqueuses ou les Pénélope (pp. 21-74), e si occupa essenzialmente di Delphine de Girardin e del genere da lei inventato, ripercorrendo la storia di quest'ultimo sino alle sue trasformazioni negli anni della Belle Époque; il secondo capitolo, Les publicistes ou les Cassandre (pp. 75-122), studia principalmente George Sand e Marie d'Agoult, ma anche Juliette Adam e Claude Vignon; il terzo, Les Frondeuses ou les Bradamante (pp. 123-176), presenta da un lato la reporter Séverine interrogandosi sul suo statuto di prima giornalista professionista, dall'altro Marguerite Durand, modello contrapposto, che fondò il primo quotidiano femminile, $L a$ Fronde, e poi le giornaliste al fronte, tra le quali già va annoverata la 
grande Colette; il quarto capitolo è dedicato a Les avanturières ou les amazones (pp. 177-224), figure come quelle di Isabelle Eberhardt, Alexandra David-Néel e Ella Maillart, indirizzate al giornalismo etnografico; il quinto, Les rédactrices professionnelles ou les Sappho (pp. 225-288), approda al xx secolo offrendo un'inchiesta sulle condizioni socioeconomiche del mestiere dal punto di vista femminile e dedicando un'intera sezione a colei che forse più di ogni altra scrittrice professionista ha praticato il giornalismo come parte integrante e determinante del suo lavoro, Colette; infine il sesto capitolo studia Les grandes reporters ou les Dalila (pp. 289-358), illustrando il genere del grande reportage a scala planetaria realizzato da giornaliste importanti e spesso dimenticate come Titaÿna, Simone Téry e Andrée Viollis. La Conclusion (pp. 359-374), oltre a tirare le fila del discorso, apre la prospettiva sul dopo rispetto al periodo studiato, sulla seconda metà del xx secolo e le grandi giornaliste che lo hanno caratterizzato. Arricchiscono il volume un'ampia Bibliographie sélective (pp. 375-386) e un ricco e suggestivo inserto fotografico. 